\title{
Las concesiones forestales de castaña de la Amazonía peruana ¿un éxito o un fracaso? ${ }^{1}$
}

\author{
Hélène V Willemª, Verina J Ingram y Manuel R Guariguatab
}

\section{Mensajes principales}

- En el Departamento de Madre de Dios, Perú, el desempeño de las concesiones para la extracción de castaña, establecidas en el año 2000, ha sido contrastante tanto dentro (Reserva Nacional Tambopata) como fuera de áreas protegidas.

- Sobre la base de un conjunto de indicadores de gobernanza, los resultados sugieren que las concesiones de castaña dentro de la Reserva tienen un mejor desempeño que las que están fuera de ella. Fuera de áreas protegidas, donde se concentra la gran mayoría de las concesiones, se encontró una situación paradójica de exceso de regulación, pero mínima intervención por parte de las agencias estatales; monitoreo y sanciones inefectivas; poco cumplimiento de la ley con medidas punitivas excesivamente altas y desbalances de poder en la cadena de valor.

- La sostenibilidad a largo plazo del sistema de concesiones de castaña podría estar comprometida. Una nueva visión requiere el reconocimiento formal de la variedad de usos de la tierra -particularmente fuera de áreas protegidas- la implementación y validación de enfoques silviculturales multiuso, una gestión más en línea con las aspiraciones locales y el establecimiento de plataformas de negociación efectivas y eficaces con diferentes sectores productivos y agencias gubernamentales.

En términos amplios, la gobernanza forestal exitosa puede definirse como el ejercicio de autoridad y el desarrollo de instituciones que garanticen el manejo forestal sostenible mediante el mantenimiento de los valores, estructura ecosistémica y función del bosque, a la vez que se satisfacen las necesidades humanas. Las concesiones forestales han demostrado ser un modelo popular de uso de la tierra en áreas remotas con baja

\footnotetext{
1 Traducido y modificado del artículo: Willem, H., Ingram, V.J., Guariguata, M. R. 2019. Brazil nut concessions in the Peruvian Amazon: success or failure? International Forestry Review 21:254-265

a Grupo de Políticas de Conservación de los Bosques y la Naturaleza, Universidad de Wageningen, Países Bajos b Centro para la Investigación Forestal Internacional, Lima, Perú
}

densidad de población y una limitada intervención por parte de las autoridades forestales (Karsenty et al. 2008). Esto involucra el otorgar tierras forestales públicas, junto con los recursos que contienen, a través de un contrato a largo plazo que permita la explotación de madera y/o de productos forestales no maderables (PFNM) a una empresa privada, una comunidad, un individuo o una organización no gubernamental, y sujeto al cumplimiento de un conjunto específico de actividades de gestión y salvaguardas (Balbinotto et al. 2012).

A pesar de que el modelo de concesión forestal ha sido implementado a nivel mundial, se encuentra principalmente en regiones tropicales. Sin embargo, el modelo de concesión forestal ha sido criticado por sus recurrentes "fallas de diseño" (FAO 2018). Y, 
entre otras falencias, por permitir la corrupción y promover a la vez la tala ilegal (Finer et al. 2014); por estar regulado oficialmente con base en ciclos de corte de madera poco realistas con el fin de mantener rendimientos sostenibles (McPherson et al. 2012); por la carencia de monitoreo adecuado que incentive el manejo adaptativo (Balbinotto et al. 2012); por su fracaso en la solución de temas de derechos a la tierra que se superponen (Karsenty 2016); y por no siempre reconocer formalmente los múltiples usos del bosque y las aspiraciones locales (Lescuyer et al. 2015).

Aunque las concesiones forestales han sido promovidas principalmente para la explotación maderera, el modelo ha sido aplicado al aprovechamiento de PFNM, como por ejemplo las Unidades de Asignación de Permisos de pygeum (Prunus africana) en Camerún (Ingram 2014) $y$, en Guatemala, las concesiones comunitarias para el aprovechamiento de madera y PFNM (Radachowsky et al. 2012). En Perú, donde se ubica el presente estudio, la recolección de castaña (proveniente de los frutos del árbol amazónico, Bertholletia excelsa), un PFNM emblemático de la cuenca amazónica, se realiza en concesiones autorizadas por el Gobierno desde el año 2000 cuando se hicieron respetar los derechos adquiridos que los recolectores de castaña habían obtenido en contratos de extracción ya existentes. Desde entonces, a nuestro entender, no se han realizado evaluaciones detalladas de la efectividad de las concesiones de castaña en cuanto a los beneficios sociales y ambientales, a pesar de la importancia de este PFNM en la economía rural y de su papel en promover la conservación del bosque (Guariguata et al. 2017). Independientemente del objetivo de la gestión de la concesión (p. ej. producción de madera, PFNM, recreación, conservación), una evaluación del desempeño del modelo de concesiones forestales debería incluir tanto las instituciones de gobierno (Balbinotto et al. 2012) como el contexto sociopolítico (Singer y Karsenty 2008). Un análisis de ambas dimensiones son el centro de este estudio.

\section{Las concesiones de castaña en Perú}

Las concesiones de castaña en el departamento de Madre de Dios en Perú, se otorgan de acuerdo a dos mecanismos contrastantes: las que se localizan dentro de áreas protegidas (en este estudio, a las situadas en la Reserva Nacional Tambopata se les mencionará como "la Reserva") y las que se localizan fuera de áreas protegidas (Peña 2010). En este estudio se contrastan estos dos tipos de tenencia de acuerdo a la predicción de que se traducirían en diferentes resultados en cuanto a la gobernabilidad. La recolección de castaña, considerada como un uso forestal de bajo impacto, se permite en todo tipo de bosque público en Perú. Las entidades que otorgan las concesiones de castaña son el Servicio Nacional de Áreas Naturales Protegidas (SERNANP) en áreas protegidas y la Dirección Regional Forestal y de Fauna Silvestre (DRFFS) en el resto del país. Para el año 2017, de acuerdo con la DRFFS, en Madre de Dios había 1123 concesiones de castaña registradas fuera de áreas protegidas, en tanto que el SERNANP reportó 85 concesiones en la Reserva y 13 en la zona de amortiguamiento del Parque Nacional Bahuaja Sonene, cerca de la Reserva.

\section{El proceso de otorgamiento de concesiones de castaña}

Las regulaciones para la explotación de recursos forestales en Perú surgieron por primera vez con la Ley Forestal No. 21147 (1975). Esta ley no consideró criterios de sostenibilidad, lo que llevó casi a la extinción local de varias especies de madera. En respuesta, el Gobierno creó el modelo de concesión actual para promover el uso sostenible del bosque (Smith et al. 2006).

Cuando fueron establecidas, las concesiones de castaña se registraron solo para la recolección del fruto, aun cuando tales bosques albergaban volúmenes sustanciales de especies maderables de valor medio y alto (Cossío et al. 2011). Durante el proceso de asignación de concesiones, se dio prioridad a los castañeros que pudieran demostrar un nexo económico y social con la tierra que solicitaban (Cossío et al. 2011), aunque el proceso de delimitación se consideró poco coordinado, con fondos insuficientes e impreciso en el aspecto espacial (Chávez et al. 2012). De acuerdo con la DRFFS, el área de concesiones individuales de castaña registrada en la actualidad fuera de áreas protegidas varía entre 24 ha y 4373 ha (promedio: 860 ha) mientras que el área total de concesiones de castaña en Madre de Dios se estima en 1 millón ha (Perales y Guariguata 2015).

\section{La gobernanza en las concesiones de castaña en Madre de Dios}

Fuera de la Reserva, la DRFFS tiene funciones administrativas, en tanto que el monitoreo de la concesión y el cumplimiento de las regulaciones está a cargo del Organismo de Supervisión de los Recursos Forestales y de Fauna Silvestre (OSINFOR). Dentro de la Reserva, el SERNANP solo es responsable de supervisar las concesiones. A pesar de que los concesionarios de castaña tienen derechos exclusivos sobre sus concesiones, 


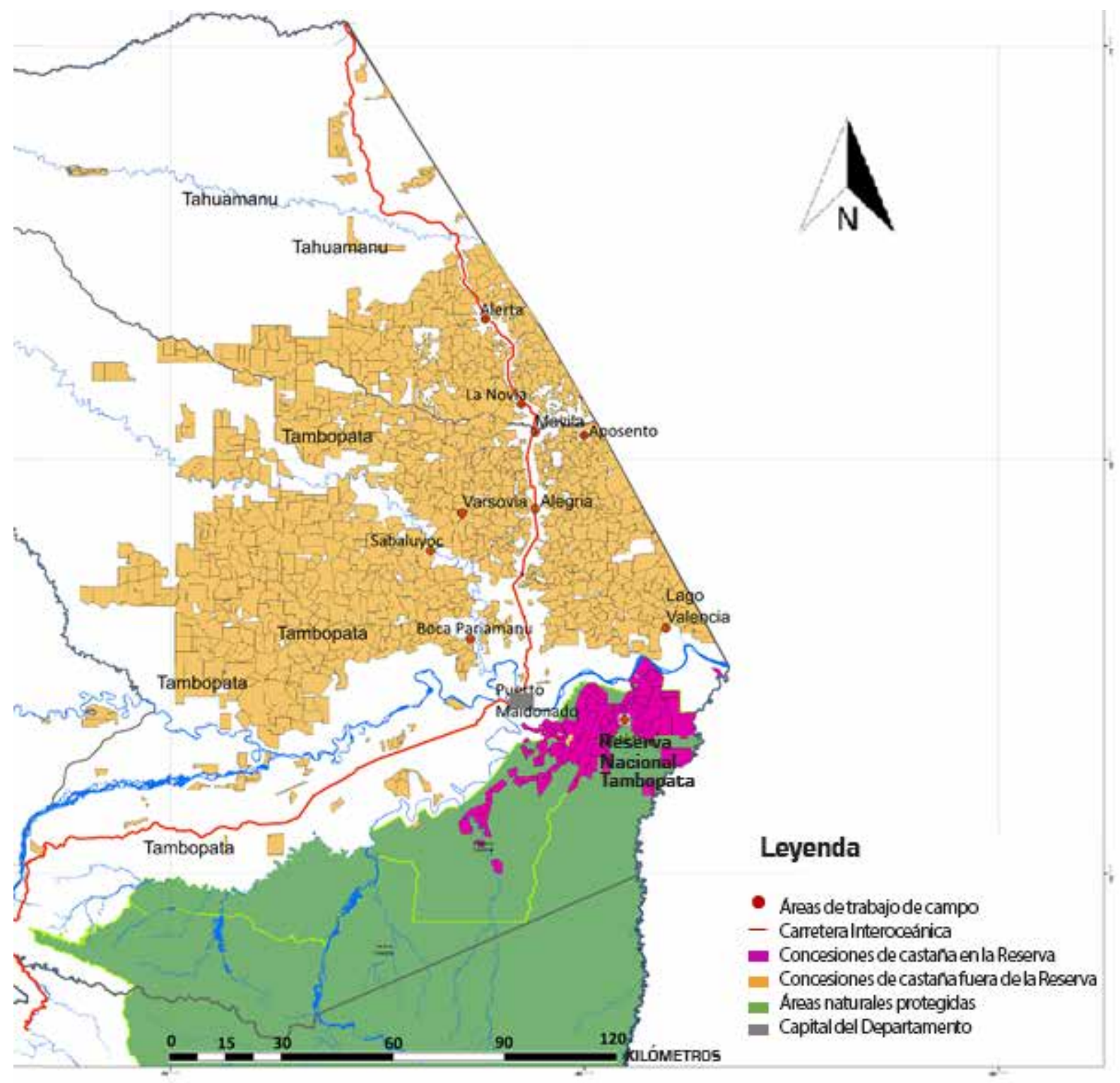

Figura 1. Localización de las concesiones de castaña dentro de la Reserva Nacional Tambopata (en color fucsia) y fuera de áreas protegidas (en color amarillo), Departamento de Madre de Dios, Amazonía peruana.

Nota: Los nombres de las áreas de trabajo de campo (puntos de color rojo) son los empleados por los concesionarios entrevistados

la tala de árboles de castaña es ilegal (Cossío et al. 2011). Fuera de áreas protegidas, los castañeros tienen derechos de enajenación para transferir la concesión a un tercero, aunque no pueden venderla. Una vez recolectan las castañas del piso del bosque, los castañeros están obligados a pagar un impuesto de recolección por kilo a la DRFFS, en tanto que los castañeros en concesiones dentro de la Reserva pagan tal impuesto al SERNANP. Hasta su abolición en el año 2014, los castañeros de concesiones ubicadas fuera de la Reserva tenían que elaborar un Plan Operativo Anual (POA) para cosechar (Perales y
Guariguata 2015). Luego, la Ley Forestal No. 29763 (SERFOR 2015) buscó simplificar las regulaciones sobre la recolección de castaña. El POA fue reemplazado por una Declaración de Manejo (DEMA) por un período renovable de hasta cinco años. La diferencia principal entre estos dos requisitos es que la DEMA la firma directamente el concesionario y no a través de un funcionario de gobierno, como se hacía con el POA. A partir del año 2004, a los concesionarios ubicados fuera de la Reserva se les ha permitido aprovechar madera luego de elaborar un Plan de Manejo Forestal Intermedio, que en comparación 
con los elaborados en las concesiones madereras vecinas tienen mínimos requisitos burocráticos y fiscales (Cossío et al. 2011). La aprobación de estos planes requiere la inspección y aprobación en campo por parte de un funcionario forestal.

Una diferencia clave para los concesionarios de castaña dentro de la Reserva radica en que no se les permite cosechar madera. Adicionalmente, la forma en que las cantidades de castaña se manejan y se reportan a las autoridades relevantes, es diferente para las concesiones dentro de la Reserva y para las que están fuera de esta. Las concesiones fuera de la Reserva (las cuales, como se mencionó, son la gran mayoría en Madre de Dios) deben calcular, antes de cosechar, el volumen que probablemente se extraerá en ese año (cálculo que se hace con el número de árboles de castaña en cada concesión) y reportar este valor a la DRFFS, mientras que las concesiones dentro de la Reserva solo reportan el volumen extraído a las autoridades luego de que ha terminado la cosecha (Perales y Guariguata 2015). Las concesiones de castaña en la Reserva se encuentran reguladas por el Plan Maestro de la Reserva Nacional Tambopata y el Parque Nacional Bahuaja Sonene. Este plan renovable a cinco años define la regulación de todas las actividades dentro de áreas protegidas. En contraste con las concesiones fuera de las áreas protegidas, las que se ubican al interior de la Reserva solo pueden accederse durante la época de preparación para la cosecha (15 días entre los meses de noviembre y diciembre) y durante la estación de cosecha (hasta abril). Cualquier visita adicional requiere de autorización por parte del SERNANP.

\section{Métodos}

Para evaluar de qué manera el sistema de concesiones de castaña se ha desempeñado luego de más de una década de su implementación, el análisis se enmarcó mediante el enfoque de Von Halle (2014), en el que los factores que influyen en los resultados de gobernanza forestal se agrupan en tres componentes esenciales: (i) los mecanismos institucionales, (ii) las características de los usuarios del bosque, y (iii) las características de la cadena de valor del producto forestal (tabla 1). Este marco asume que, en caso de no haber acciones orientadas a resolver los problemas existentes de cualquiera de los componentes antes mencionados, los resultados de gobernanza serán subóptimos, y por lo tanto se verá en peligro el uso sostenible del bosque. Con base en una revisión de la literatura y guiados por los tres componentes descritos anteriormente, se definió un conjunto de condiciones habilitantes e indicadores respectivos para evaluar el desempeño de las concesiones de castaña en Perú en cuanto a su capacidad para mantener los valores socioeconómicos y ambientales, a la vez que promueven el uso sostenible del bosque (tabla 1).

Como fuente principal de información, se realizaron 50 entrevistas semiestructuradas entre los meses de mayo y julio de 2017 con los concesionarios (39 concesiones fuera de áreas protegidas y 11 dentro de la Reserva). Ante la ausencia de disponibilidad de listas con información de contacto sobre las concesiones, y debido a que algunos concesionarios no estaban dispuestos a ser entrevistados, se utilizó el método de la bola de nieve. Para tal fin, se contactaron a los líderes de las asociaciones de castañeros. Para minimizar el sesgo y maximizar la representatividad, se llevaron a cabo entrevistas en diferentes lugares (figura 1), mientras se trataba de lograr un equilibrio entre las concesiones de castaña cercanas a la Carretera Interoceánica (18) y las que se encuentran más alejadas (21). Los cuestionarios se adaptaron a las diferencias en las regulaciones y a las autoridades encargadas de la supervisión tanto dentro como fuera de las áreas protegidas, como se describió anteriormente. Las preguntas cubrieron aspectos normativos y regulatorios, problemas de seguridad de tenencia, interacciones con agencias estatales, el grado de participación en asociaciones de productores de castaña y relaciones con partes interesadas relevantes a lo largo de cadenas de valor de castaña (y de madera), de acuerdo a los indicadores 1 a 10 (tabla 1). Adicionalmente, se sostuvieron entrevistas con dos funcionarios del gobierno central y tres funcionarios del gobierno local, y se realizó una entrevista grupal en la oficina local de OSINFOR. Además, se entrevistó a un oficial local del SERNANP, a tres individuos de una organización de donantes privados y al personal de organizaciones no gubernamentales locales. Finalmente, se hicieron entrevistas a dos representantes de las empresas procesadoras de castaña (una certificada y otra no certificada).

El contenido de los datos oficiales secundarios fue analizado más a fondo; específicamente, datos de OSINFOR sobre el cumplimiento por parte de los concesionarios de las normas y regulaciones que rigen la recolección de castaña y el uso del bosque. También se analizaron los informes anuales de la DRFFS y del SERNANP sobre reclamos y quejas presentadas por los concesionarios a las autoridades pertinentes, así como datos sobre los volúmenes anuales de castaña 
Tabla 1. Componentes esenciales y condiciones habilitantes de gobernanza (tomado de Von Halle 2014) e indicadores de desempeño, relacionados para la evaluación del sistema de concesiones de castaña.

\begin{tabular}{|c|c|c|c|}
\hline $\begin{array}{l}\text { Componentes } \\
\text { esenciales }\end{array}$ & $\begin{array}{l}\text { Condicioens } \\
\text { habilitantes de la } \\
\text { gobernanza }\end{array}$ & Indicador & Fuente \\
\hline \multirow[t]{6}{*}{$\begin{array}{l}\text { Mecanismos } \\
\text { institucionales }\end{array}$} & \multirow[t]{2}{*}{$\begin{array}{l}\text { Instituciones que se } \\
\text { ajustan al contexto } \\
\text { local }\end{array}$} & $\begin{array}{l}\text { 1. Ausencia de carga } \\
\text { regulatoria para los } \\
\text { concesionarios }\end{array}$ & $\begin{array}{l}\text { Agrawal (2007), Mayers y } \\
\text { Vermeulen (2002), Ros-Tonen y } \\
\text { Kusters (2011), Tucker (2010) }\end{array}$ \\
\hline & & $\begin{array}{l}\text { 2. Inclusión de los } \\
\text { concesionarios de castaña en el } \\
\text { proceso de toma de decisiones }\end{array}$ & $\begin{array}{l}\text { Agrawal et al. (2008), Mayers y } \\
\text { Vermeulen (2002), Ros-Tonen y } \\
\text { Kusters (2011), Tucker (2010) }\end{array}$ \\
\hline & \multirow[t]{2}{*}{$\begin{array}{l}\text { Calidad de la } \\
\text { aplicación de la ley }\end{array}$} & $\begin{array}{l}\text { 3. Monitoreo realizado } \\
\text { regularmente }\end{array}$ & $\begin{array}{l}\text { Gibson et al. (2005), Gray (2002), } \\
\text { Tucker (2010) }\end{array}$ \\
\hline & & $\begin{array}{l}\text { 4. Efectividad de las medidas } \\
\text { y de las herramientas para } \\
\text { prevenir los delitos forestales }\end{array}$ & $\begin{array}{l}\text { Azevedo-Ramos et al. (2015), } \\
\text { Ferraz y Seroa da Motta (1998), } \\
\text { Kishor y Rosenbaum (2012) }\end{array}$ \\
\hline & \multirow[t]{2}{*}{$\begin{array}{l}\text { Derechos de } \\
\text { tenencia }\end{array}$} & $\begin{array}{l}\text { 5. Existencia y efectividad de } \\
\text { mecanismos para la resolución } \\
\text { de disputas y conflictos de } \\
\text { tenencia y de derechos }\end{array}$ & $\begin{array}{l}\text { Agrawal et al. (2008), Kishor y } \\
\text { Rosenbaum (2012), Mayers y } \\
\text { Vermeulen (2002), Von Halle } \\
\text { (2014) }\end{array}$ \\
\hline & & $\begin{array}{l}\text { 6. Límites definidos con } \\
\text { claridad }\end{array}$ & $\begin{array}{l}\text { Gray (2002), Mayers y Vermeulen } \\
\text { (2002), Ostrom (2009), Tucker } \\
(2010)\end{array}$ \\
\hline \multirow[t]{3}{*}{$\begin{array}{l}\text { Usuarios } \\
\text { (concesionarios de } \\
\text { castaña) }\end{array}$} & \multirow[t]{2}{*}{$\begin{array}{l}\text { El valor del recurso } \\
\text { merece una gestión } \\
\text { sostenible }\end{array}$} & $\begin{array}{l}\text { 7. Dependencia de los } \\
\text { concesionarios de los medios } \\
\text { de vida provenientes de los } \\
\text { recursos de las concesiones de } \\
\text { castaña }\end{array}$ & Agrawal (2001), Ostrom (2009) \\
\hline & & $\begin{array}{l}\text { 8. Percepción de los } \\
\text { concesionarios del valor de las } \\
\text { concesiones de castaña }\end{array}$ & Agrawal (2001), Ostrom (2009) \\
\hline & $\begin{array}{l}\text { Liderazgo de los } \\
\text { usuarios del bosque } \\
\text { y su participación en } \\
\text { el proceso de toma } \\
\text { de decisiones }\end{array}$ & $\begin{array}{l}\text { 9. Participación en asociaciones } \\
\text { de recolectores de castaña y } \\
\text { sus beneficios }\end{array}$ & $\begin{array}{l}\text { Agrawal (2001), Marshall et al. } \\
\text { (2006), Mayers y Vermeulen } \\
\text { (2002), Ostrom (2009), Ros-Tonen y } \\
\text { Kusters (2011) }\end{array}$ \\
\hline $\begin{array}{l}\text { Cadena de valor } \\
\text { del producto } \\
\text { forestal }\end{array}$ & $\begin{array}{l}\text { Oportunidades } \\
\text { de mercado que } \\
\text { permiten que los } \\
\text { concesionarios se } \\
\text { beneficien de los } \\
\text { recursos forestales } \\
\text { de manera } \\
\text { sostenible }\end{array}$ & $\begin{array}{l}\text { 10. Ausencia de barreras para } \\
\text { entrar al mercado }\end{array}$ & $\begin{array}{l}\text { Marshall et al. (2006), Mayers y } \\
\text { Vermeulen (2002), Ros-Tonen y } \\
\text { Kusters (2011), Von Halle (2014) }\end{array}$ \\
\hline
\end{tabular}


recolectados y la emisión de permisos de extracción de madera en concesiones fuera de la Reserva.

\section{Resultados}

\section{Indicador 1. Carga regulatoria}

La mayoría (72\%) de los 39 concesionarios que se ubican fuera de la Reserva sostuvieron que las regulaciones que rigen las concesiones, en su conjunto, no son legítimas. En particular, perciben que las regulaciones sobre la extracción de madera y la prohibición de la remoción de tierras para la agricultura son demasiado estrictas. Con respecto a la extracción de madera, más de la mitad de las 39 concesiones informaron que no recolectan madera porque obtener un permiso es demasiado costoso, es complicado en el procedimiento y requiere mucho tiempo. Según OSINFOR, los concesionarios con frecuencia firman permisos de aprovechamiento de madera sin entender a cabalidad el contenido de este, ya que usualmente estas formas son llenadas por madereros que actúan como tercera parte. Como resultado 14 (36\%) de los 39 concesionarios entrevistados, fueron amonestados con multas por violar los términos de los permisos de cosecha de madera. Además, más de la mitad (59\%) de los concesionarios entrevistados fuera de la Reserva consideraron que las normas y regulaciones actuales desincentivan el manejo forestal sostenible. La imposición de multas por parte de OSINFOR aparentemente ha conllevado a la reducción de peticiones de permiso para la extracción de madera en concesiones fuera de la Reserva. Aunque no se puede descartar que al mismo tiempo los rendimientos de madera en pie hayan disminuido en las concesiones, lo cual hace la extracción menos atractiva, el número de permisos de extracción de madera otorgados se redujo de 300 en 2014 a 97 en 2015 y aumentó ligeramente a 99 en 2016. A diferencia de lo anterior, los concesionarios dentro de la Reserva no identificaron grandes cargas regulatorias o administrativas relacionadas con la gestión de la concesión. Esencialmente, deben hacer menos trámites, en tanto que los requisitos legales están bien alineados con las realidades locales de las actividades de recolección de castaña. Como se mencionó, los concesionarios fuera de la Reserva deben calcular e informar a la DRFFS los volúmenes de castaña que cosecharán (algo cuya utilidad no es obvia en términos de mejora del manejo; Perales y Guariguata 2015), mientras que, dentro de la Reserva, los concesionarios solo deben informar los volúmenes reales de castaña cosechada al SERNANP sin adelantar ningún trámite.

\section{Indicador 2. Inclusión de los concesionarios de castaña en los procesos de toma de decisiones}

Fuera de la Reserva, $30 \%$ de los 39 concesionarios entrevistados sostuvieron que OSINFOR funge como controlador y brinda poco, o casi ningún, apoyo a la mejora de prácticas de manejo o a la inclusión de concesionarios en la toma de decisiones locales. El $62 \%$ restante no opinó sobre OSINFOR, aunque algunos entrevistados reconocieron que dicho organismo había organizado anteriormente talleres. En contraste, los concesionarios ubicados dentro de la Reserva indicaron que el SERNANP es más un aliado, que una agencia de mando y de control. Durante la elaboración del Plan Maestro (elaborado cada cinco años), el SERNANP organiza talleres participativos con los concesionarios de castaña, para incluir recomendaciones y observaciones que puedan ayudar a mejorar la gestión de las concesiones. Sin embargo, debe recalcarse que para el SERNANP puede ser más fácil trabajar con un número limitado de concesionarios en la Reserva (cerca de 80 en total), en tanto que OSINFOR debe lidiar con más de 1000 concesiones que se encuentran dispersas fuera de la Reserva.

\section{Indicador 3. Monitoreo hecho con regularidad}

Los entrevistados del SERNANP y del OSINFOR sostuvieron que no tenían suficiente personal para monitorear de forma regular las concesiones. Al momento de este estudio, OSINFOR solo tenía seis funcionarios asignados a 1123 concesiones para la inspección anual. Entre los años 2009 y 2016, OSINFOR realizó 634 inspecciones de concesiones de castaña ubicadas fuera de la Reserva, lo que sugiere que cerca de la mitad de las concesiones no han sido jamás inspeccionadas. En contraste, y de acuerdo con el SERNANP, al interior de la Reserva los funcionarios visitan anualmente cerca del $60 \%$ de 80 concesiones durante la época de cosecha. Aunque tales visitas no cubren cada una de las concesiones, todos los concesionarios que entran y salen de la Reserva deben someterse a un control de entrada en un momento dado. Mediante esta actividad obligatoria (la cual no se implementa fuera de la Reserva), el SERNANP puede monitorear sistemáticamente cada concesionario antes, durante y después de la estación de cosecha de castaña. 


\section{Indicador 4. Medidas y herramientas para la prevención de delitos forestales}

Del total de 634 controles de monitoreo que realizó OSINFOR fuera de la Reserva entre 2009 y 2016, $59 \%$ resultaron en sanciones, en $32 \%$ se encontró que los planes de manejo cumplían con las regulaciones existentes, y $9 \%$ no expresaron claramente si se habían cumplido con los planes de manejo, o no. La mayoría de las actividades ilegales en las concesiones ubicadas fuera de la Reserva tuvieron que ver con explotación de madera: de las 373 sanciones, la extracción de madera sin autorización fue reportada en $78 \%$ de los casos y la falsificación de planes de manejo maderero para facilitar la explotación y transporte de madera ilegal obtenida en otros lugares se reportó en 65 \% de los casos. De las 75 concesiones sometidas a inspección más de una vez por parte de OSINFOR, más de la tercera parte fueron sancionadas en las dos oportunidades. En contraste, no hubo sanciones por extracción ilegal de madera a concesiones ubicadas en la Reserva, ya que la total prohibición de esta actividad y el acceso controlado a la Reserva, pondrían fácilmente en evidencia las extracciones ilegales de madera. La mayoría de los concesionarios en la Reserva consideraron apropiado y justo el sistema de monitoreo del SERNANP.

\section{Indicador 5. Mecanismos para la resolución de disputas y conflictos sobre tenencia y derechos}

Según los documentos oficiales, las reclamaciones de los concesionarios relacionadas con la superposición de los derechos de propiedad y los límites poco claros de las concesiones, fueron financiadas directamente por los propios concesionarios en lugar de la DRFFS, que no se consideraba responsable de dichos costos. Son comunes los conflictos por robo de castaña y/o madera, y por invasión de tierra, con 22 concesiones fuera de la Reserva (de un total de 39) que mencionaron este tipo de incidentes. A pesar de que las concesiones fuera de la Reserva están obligadas a informar sobre cualquier ocurrencia de invasión de tierra a la DRFFS, y a pesar de que el contrato de las concesiones estipula que el Estado intervendría en casos de intrusión, los concesionarios sostienen que en general el proceso es costoso e inefectivo. Los concesionarios, en particular al interior de la Reserva, se quejaban por la invasión de sus concesiones. Según los entrevistados, miembros de las comunidades nativas adyacentes entran a las concesiones cuando empiezan a caer los frutos al suelo y los castañeros no han llegado a entrar a la Reserva aún. Para evitar el robo de castañas, el SERNANP dictaminó recientemente que los castañeros podían entrar a las concesiones en la Reserva antes de lo planificado.

\section{Indicador 6. Definición de límites}

Más de la mitad de las concesiones ubicadas fuera de la Reserva dijeron que los límites entre las concesiones contiguas se superponen. En la mayoría de los casos, el límite de la concesión es diferente al del área consuetudinaria antes del inicio del proceso de asignación de la concesión. En lo particular, esto crea un problema para los concesionarios que consideran los límites contractuales como una capa de derecho de propiedad formal que se posa sobre sus derechos consuetudinarios, en comparación con aquellos que solo reconocen derechos consuetudinarios o regulatorios. En contraste, la mayoría de los concesionarios en la Reserva no informaron problemas derivados de la superposición de límites.

\section{Indicador 7. Dependencia de medios de vida de concesionarios de los recursos de la concesión de castaña}

Si bien el objetivo principal de la creación de concesiones de castaña fue económico, la variabilidad en los beneficios económicos, debida a las diferentes áreas de concesión, a la facilidad de acceso, a una gran variación interanual en la producción de frutos (Rockwell et al. 2015) y a las fluctuaciones asociadas al precio de mercado, se traduce en que la castaña constituye solo una estrategia parcial como medio de vida. Sin embargo, $60 \%$ de las 18 concesiones cercanas a la Carretera Interoceánica informaron no tener fuentes de ingreso alternas. A pesar de ello, el irse a vivir a las ciudades en busca de trabajo durante la época en que no se cosecha, podría ser desventajoso dado que aumenta el riesgo de invasión de la tierra, especialmente cuando la concesión se encuentra cerca de la Carretera Interoceánica. Algunos entrevistados informaron además sobre la deforestación para agricultura en concesiones fuera de la Reserva, ya que algunos concesionarios alquilan parte de la tierra a terceros -agricultores- para obtener ingresos adicionales. Para cumplir con las regulaciones, estos mismos concesionarios posteriormente denuncian a los agricultores como invasores, ya que saben que las autoridades estatales usualmente tardan años en tomar acción. 


\section{Indicador 8. Percepción de los concesionarios sobre el valor de las concesiones de castaña}

El interés de los jóvenes en participar en la recolección de castaña parece estar disminuyendo. En general, el $28 \%$ de los 50 concesionarios entrevistados declararon que sus hijos no estaban interesados, mientras que el $41 \%$ mencionó que al menos un hijo estaba interesado. Entre los concesionarios sin hijos interesados, cinco pretenden vender la concesión a un tercero (a pesar de que esto es ilegal); insinuaron que los futuros compradores podrían estar más interesados en la madera que en los recursos no maderables. El hijo de un concesionario afirmó que para los jóvenes es un estigma ser castañero y que es más aceptable socialmente el tener un trabajo de tiempo completo en la ciudad.

\section{Indicador 9. Participación y beneficios provenientes de las asociaciones de concesionarios}

De los 50 concesionarios entrevistados, el $58 \%$ pertenece a una asociación de cosechadores de castañeros. Aquellos que no eran miembros de una asociación mencionaron desconfianza, mala gestión financiera o la influencia negativa de élites sobre los problemas de la asociación (para obtener beneficios políticos o financieros). Entre el $58 \%$ que son miembros de una asociación, el $24 \%$ informó que no obtuvo ninguna ventaja de su membresía, mientras que el $44 \%$ dijo haber obtenido una ventaja financiera. Las dos asociaciones mencionadas de haber brindado ventaja financiera, fueron Recolectores Orgánicos de la Nuez Amazónica de Perú (RONAP) y la Asociación de Castañeros de la Reserva Tambopata (ASCART). La motivación subyacente para convertirse en miembro radica en que estas asociaciones negocian mejores precios con grandes compradores.

\section{Indicador 10. Barreras de entrada al mercado}

De los 50 concesionarios que conforman la muestra de este estudio, solo $18 \%$ (que a la vez son miembros de la ASCART) dijeron tener acceso a crédito bancario, mientras que $58 \%$ han obtenido un avance de pago de algún comprador de castaña, para luego ser reembolsado con las ganancias de la cosecha. Se informó que es más probable que estos compradores individuales otorguen préstamos cuando tienen una larga relación con los concesionarios. Sin embargo, esto puede llegar a disminuir la capacidad del concesionario para cambiar a otro comprador cuando cambios en factores externos (p. ej. probables cambios en el precio de otras nueces comercializadas internacionalmente) o intrínsecos (p. ej. variación interanual en la producción de castaña) modifican el precio internacional. Adicionalmente, y dado que la estación de cosecha es corta, el valor económico de la castaña en los mercados locales tiende a incrementare gradualmente luego de la cosecha. Sin embargo, la necesidad de reembolsar los adelantos de efectivo hechos por los compradores, significa que los concesionarios generalmente se ven obligados a vender su producto justo después de que finaliza la temporada de cosecha, generalmente a un precio más bajo.

Además, como se mencionó anteriormente, los concesionarios de castaña que participan en los mercados de madera están sujetos a complejas regulaciones que los hacen muy dependientes de terceros para llevar a cabo el papeleo y ejecutar la recolección, lo que reduce en gran medida los beneficios provenientes de la explotación de madera. Finalmente, los entrevistados sostienen que los beneficios de la certificación de productos se asocian principalmente con el mejoramiento de sus habilidades en la producción sostenible y el uso del bosque, en lugar de beneficios económicos. Según un representante de una empresa de certificación, los concesionarios están menos dispuestos a adoptar la certificación porque requiere un cumplimiento más estricto de las normas, si bien no siempre se generan ventajas financieras con las prácticas no certificadas.

\section{Discusión}

Con la creación de las concesiones de castaña en el año 2000 el Gobierno peruano buscó incluir los PFNM como una manera de formalizar la diversificación del sector forestal y mejorar los medios de vida locales. Mediante un enfoque cualitativo, este estudio ha buscado dilucidar si el sistema de concesiones de castaña, a más de 15 años de su establecimiento, ha promovido el uso sostenible del bosque. El lector debe tener en cuenta que este análisis se basa en una pequeña muestra, en comparación con las más de 1000 concesiones registradas oficialmente en Madre de Dios. Por lo tanto, los resultados son de carácter indicativo y deben ser interpretados con cautela.

Al comparar las concesiones de castaña dentro de la Reserva Nacional Tambopata y aquellas fuera de áreas protegidas, las cuales constituyen la gran mayoría de las concesiones de castaña en Madre de Dios, se encontraron resultados contrastantes. Sobre la base del conjunto de indicadores aplicados en este estudio, los resultados 
sugieren que las concesiones de castaña dentro de la Reserva tienen un mejor desempeño que las que están fuera de ella. La excesiva regulación, el monitoreo ineficaz con las sanciones que lo acompañan, y la superposición de derechos de propiedad y tipos de tenencia, son problemas comunes para las concesiones fuera de las áreas protegidas, en tanto que la tala ilegal e incontrolada de madera es rampante (Praeli 2019). Cuando se combinan, estos factores pueden ser negativos para la sostenibilidad a largo plazo de las concesiones de castaña fuera de la Reserva; estos hallazgos no son muy diferentes de otras evaluaciones de la gobernanza de los PFNM en todo el mundo, en particular los sistemas que involucran tanto a los pequeños recolectores como a sus propietarios (Laird et al. 2010). En términos más generales, estos resultados reflejan problemas comunes a las concesiones de bosques tropicales a nivel mundial: gobernanza débil, reglas demasiado complicadas con un reconocimiento limitado de las aspiraciones locales en los objetivos de manejo, distribución no equitativa de beneficios y aplicación deficiente de la ley (FAO 2018). Los diferentes enfoques de gobernanza de las concesiones de castaña dentro y fuera de la Reserva por parte de diferentes agencias (la DRFFS y OSINFOR fuera de áreas protegidas, y el SERNANP dentro de la Reserva), ilustra aún más cómo los resultados de la gestión pueden contrastar incluso dentro del mismo tipo de uso forestal. Los procedimientos administrativos menos engorrosos en la Reserva parecen adecuarse a las realidades y necesidades de los recolectores de castaña, en comparación con los concesionarios fuera de la Reserva. Dicho esto, la DRFFS podría emular el enfoque participativo empleado por el SERNANP buscando promover el manejo adaptativo. También se debe tener en cuenta que, en el momento de su creación, las concesiones de castaña fuera de la Reserva no se concibieron con una mentalidad de uso múltiple, a pesar de albergar volúmenes sustanciales de madera (Cossío et al. 2011). Desde entonces, han sido gobernadas a través de enfoques mayoritariamente tecnocráticos y con una filosofía orientada a la madera (Perales y Guariguata 2015). Este problema también puede reflejar su desempeño aparentemente negativo a lo largo del tiempo.

Un mensaje clave de este análisis es que la efectividad de las medidas de comando y control es cuestionable: los concesionarios fuera de la Reserva generalmente consideraron que el Estado se ha centrado principalmente en el cumplimiento de las reglas y regulaciones, al tiempo que enfatiza las medidas punitivas, en lugar de resolver conflictos, asegurar sus derechos o ayudar a mejorar la gestión forestal. Esta situación se aplica a muchos pequeños propietarios forestales y agricultores a lo largo de toda la Amazonía (Pacheco et al. 2016). Vale la pena señalar que, en comparación con Perú, el éxito de Bolivia en dominar el mercado de exportación de castaña a nivel mundial a lo largo del tiempo, ha dependido menos de que los recolectores sigan las regulaciones y normas dirigidas por el Gobierno y más de su adaptación al mercado internacional y a las fuerzas comerciales (Guariguata et al. 2017).

El débil capital social de los concesionarios, mencionado explícitamente por los líderes de dos asociaciones de cosechadoras de castaña, es otro tema clave que obstaculiza el modelo de concesión de castaña para mejorar los valores forestales y los medios de vida locales. Ya se ha documentado un bajo grado de acción colectiva entre los recolectores de castaña en Madre de Dios; esto ha resultado en la mala gestión financiera de las asociaciones de castañeros, así como en que los recolectores son propensos a la manipulación por parte de grupos de élite para obtener ventajas financieras o políticas (Quaedvlieg et al. 2014). Esto ha hecho a los recolectores propensos a la marginación en los procesos de toma de decisiones relacionadas con los bosques, al tiempo que aumenta su vulnerabilidad ante actores poderosos a lo largo de la cadena de valor de los productos forestales (Ingram et al. 2015). Promover iniciativas de asociación merece la atención tanto de agencias gubernamentales como de organizaciones no gubernamentales. El colectivismo tanto en el procesamiento de castaña como en las acciones también podría ayudar a estimular la comercialización del producto en el mercado nacional. Por ejemplo, en Brasil parece haber una gama más amplia de productos de castaña principalmente para el mercado nacional (Homma et al. 2014) en comparación con Perú y Bolivia, los cuales se orientan principalmente a los mercados de exportación de castaña con y sin cáscara, aunque con signos incipientes de diversificación del producto.

Sobre el tema de la integración de objetivos de manejo, esta investigación no encontró evidencia clara de un esfuerzo concertado, eficaz, multisectorial y de múltiples actores en las concesiones de castaña, particularmente fuera de las áreas protegidas. Las acciones concretas para integrar, por ejemplo, agricultura, buenas prácticas silvícolas, extracción de madera y castaña, y ecoturismo, como un modelo holístico de uso de la tierra, no fueron evidentes durante la investigación. Aunque se sabe bien que cuando se involucra más de un uso (y más de un actor), los arreglos necesarios para balancear 
aspectos negativos y positivos de la gestión forestal son más exigentes con respecto a la explotación de un solo producto (Guariguata et al. 2010), existe evidencia de que, en Madre de Dios, los enfoques de gestión de uso múltiple pueden ser viables. Tanto Nunes et al. (2012) y Kirby et al. (2010) calculan que, de asociarse con actividades de ecoturismo y/o explotación maderera sostenible, la recolección de castaña en Madre de Dios podría competir económicamente con agricultura de pequeña escala. Otro estudio en Madre de Dios con base científica sugiere que, a bajas intensidades de aprovechamiento de madera, la producción de frutos de los árboles de castaña no se afectaría (Rockwell et al. 2015).

En conclusión, si se busca que el sistema de concesiones de castaña (particularmente fuera de áreas protegidas) entre en una siguiente fase, esto podrá lograrse solo mediante el reconocimiento formal de los múltiples usos de la tierra, la implementación y validación de enfoques de manejo múltiple del bosque, una gestión más en línea con las aspiraciones locales y el establecimiento de plataformas de negociación efectivas y eficaces con diferentes sectores productivos y agencias gubernamentales.

\section{Referencias}

Agrawal A. 2001. Common property institutions and sustainable governance of resources. World Development 29 (10): 1649-72.

Agrawal A. 2007. Forests, governance, and sustainability: Common property theory and its contributions. International Journal of the Commons 1 (1): 111-36. https://doi.org/10.18352/ijc.10.

Agrawal A, Chhatre A y Hardin R. 2008. Changing governance of the world's forests. Science 320 (5882): 1460-62.

Azevedo-Ramos C, Silva JNM y Merry F. 2015. The evolution of Brazilian forest concessions. Elementa: Science of the Anthropocene 3. https://doi.org/10.12952/ journal.elementa.000048.

Balbinotto G, Tillmann EA y Ratnieks I. 2012. Regulation and moral hazard in forest concessions in Brazil. Berkeley Program in Law and Economics, UC Berkeley, Berkley, United States of America. https://escholarship.org/uc/ item/88h6m8sw, consultado el 12 de marzo de 2019.

Chávez A, Guariguata MR, Cronkleton P, Menton M, Capella JL, Araujo JP y Quaedvlieg J. 2012. Superposición espacial en la zonificación de bosques en Madre de Dios: Implicaciones para la sostenibilidad del recurso castañero. Infobrief No. 58. Centro para la Investigación Forestal Internacional (CIFOR), Bogor, Indonesia.
Cossío-Solano RE, Guariguata MR, Menton M, Capella JL, Ríos L. y Peña P. 2011. El aprovechamiento de madera en las concesiones castañeras (Bertholletia excelsa) en Madre de Dios, Perú: Un análisis de su situación normativa. Working Paper No. 56. Centro para la Investigación Forestal Internacional (CIFOR), Bogor, Indonesia.

[FAO]. Organización de las Naciones Unidas para la Alimentación y la Agricultura2018. Making forest concessions in the tropics work to achieve the 2030 Agenda: Voluntary Guidelines. Forestry Paper No. 180. Organización de las Naciones Unidas para la Alimentación y la Agricultura (FAO), Roma, Italia.

Ferraz C. y Serôa da Motta R. 1998. Economic incentives and forest concessions in Brazil. Planejamento Políticas Públicas 18: 259-86.

Finer M, Jenkins C, Sky M. y Pine J. 2014. Logging concessions enable illegal logging crisis in the Peruvian Amazon. Scientific Reports 4 (1): 719. https://doi. org/10.1038/srep04719.

Gibson CC, Williams JT y Ostrom E. 2005. Local enforcement and better Forests. World Development 33: $273-284$.

Gray JA. 2002. Forest concession policies and revenue systems: Country experience and policy changes for sustainable tropical forestry. Banco Mundial, Washington, D.C.

Guariguata MR, García-Fernández C, Sheil D, Nasi R, Herrero-Jauregui C, Cronkleton P y Ingram V. 2010. Compatibility of timber and non-timber forest product management in natural tropical forests: Perspectives, challenges, and opportunities. Forest Ecology and Management 259 (3): 237-245.

Guariguata MR, Cronkleton P, Duchelle AE y Zuidema PA. 2017. Revisiting the 'cornerstone of Amazonian conservation': A socioecological assessment of Brazil nut exploitation. Biodiversity and Conservation 26 (9): 2007-2027.

Homma AKO, Menezes AJEA y Maués MM. 2014. Castanheira-do-pará: os desafios do extrativismo para plantios agrícolas. Boletin do Museu Paraense Emilio Goeldi. Ciencias Naturais 9: 293-306.

Ingram VJ. 2014. Win-wins in forest product value chains: How governance impacts the sustainability of livelihoods based on non-timber forest products from Cameroon. Centro de Estudios Africanos, Leiden, Países Bajos.

Ingram V, Ros-Tonen M y Dietz T. 2015. A fine mess: Bricolaged forest governance in Cameroon. International Journal of the Commons 9 (1): 41-64.

Karsenty A. 2016. The contemporary forest concessions in West and Central Africa: Chronicle of a foretold decline? Forestry Policy and Institutions Working Paper No. 34. 
Organización de las Naciones Unidas para la Alimentación y la Agricultura (FAO), Roma, Italia.

Kirby CA, Giudice-Granados R, Day B, Turner K, VelardeAndrade L, Dueñas-Dueñas A. et al. 2010. The market triumph of ecotourism: An economic investigation of the private and social benefits of competing land uses in the Peruvian Amazon. PLoS ONE 5 (9): e13015. https://doi. org/10.1371/journal.pone.0013015.

Kishor N y Rosenbaum K. 2012. Assessing and monitoring forest governance: A user's guide to a diagnostic tool. Banco Mundial, Washington D.C. http://documents.worldbank. org/curated/en/196281468337192253/Assessing-andmonitoring-forest-governance-a-users-guide-to-adiagnostic-tool, consultado el 12 de marzo de 2019.

Laird S, Ingram V, Awono A, Ndoye O, Sunderland T, Lisinge E y Nkuinkeu R. 2010. Integrating customary and statutory systems: The struggle to develop a legal and policy framework for NTFPs in Cameroon. En: Laird SA, McLain RJ y Wynberg RP. (eds.) Wild product governance: Finding policies that work for non-timber forest products. Earthscan, Londres. 53-70 pp.

Lescuyer G, Mvongo-Nkene MN, Monville G, Elanga-Voundi MB y Kakundika T. 2015. Promoting multiple-use forest management: which tradeoffs in the timber concessions of Central Africa? Forest Ecology and Management 349: 20-28.

Marshall E, Schreckenberg K y Newton AC. 2006. Commercialization of non-timber forest products: Factors influencing success. Lessons learned from Mexico and Bolivia and policy implications for decision-makers. UNEP-WCMC Biodiversity Series No. 23. United Nations Environment Programme: World Conservation Monitoring Centre, Cambridge. 136 pp.

Mayers J y Vermeulen S. 2002. Power from the trees: How good forest national governance can help reduce poverty. Opinion: World Summit on Sustainable Development. International Institute for Environment and Development (IIED), Londres.

McPherson AJ, Carter DR, Schulze MD, Vidal E y Lentini MW.2012. The sustainability of timber production from Eastern Amazonian forests. Land Use Policy 29: 339-350. Nunes F, Soares-Filho B, Giudice R, Rodrigues H, Bowman M, Silvestrini R y Mendoza E. 2012. Economic benefits of forest conservation: Assessing the potential rents from Brazil nut concessions in Madre de Dios, Peru to channel REDD+ investments. Environmental Conservation 39 (2): 132-143.

Ostrom E. 2009. A general framework for analyzing sustainability of social-ecological systems. Science 325 (5939): 419-22.
Pacheco P, Mejía E, Cano W y de Jong W. 2016. Smallholder forestry in the Western Amazon: Outcomes from forest reforms and emerging policy perspectives. Forests 7 (9).

Peña P. 2010. La castaña y la shiringa en Madre de Dios: Análisis del marco legal y propuestas participativas para su mejora. Sociedad Peruana de Derecho Ambiental, Lima, Perú.

Perales E y Guariguata M. 2015. ¿Qué dicen los números? Consideraciones para una simplificación normativa del aprovechamiento y transporte de la castaña en Madre de Dios. Infobrief No. 117. Center for International Forestry Research (CIFOR), Bogor, Indonesia.

Praeli Y S. 2019. Madre de Dios: Seven Brazil nut concessions investigated for illegal timber extraction. Mongabay. https://news.mongabay.com/2019/02/ madre-de-dios-seven-brazil-nut-concessionsinvestigated-for-illegal-timber-extraction;/, consultado el 12 de marzo de 2019.

Quaedvlieg J, Roca MG. y Ros-Tonen MA. 2014. Is Amazon nut certification a solution for increased smallholder empowerment in Peruvian Amazonia? Journal of Rural Studies 33: 41-55.

Radachowsky J, Ramos VH, McNab R, Baur EH y Kazakov N. 2012. Forest concessions in the Maya Biosphere Reserve, Guatemala: A decade later. Forest Ecology and Management 268: 18-28. https://doi.org/10.1016/j. foreco.2011.08.043.

Rockwell CA, Guariguata MR, Menton M, Arroyo Quispe E, Quaedvlieg J, Warren-Thomas E, et al. 2015. Nut production in Bertholletia excelsa across a logged forest mosaic: Implications for multiple forest use. PLOS ONE 10 (8): e0135464.

Ros-Tonen MA. y Kusters K. 2011. Pro-poor governance of non-timber forest products: The need for secure tenure, the rule of law, market access and partnerships. En: Non-timber forest products in the global context. Springer Berlin, Heidelberg, Alemania. 189-207 pp.

SERFOR. 2015. Ley forestal y de fauna silvestre no. 29763 y sus reglamentos. Servicio Nacional Forestal y de Fauna Silvestre (SERFOR). Lima, Perú.

Singer B y Karsenty A. 2008. Can concessions be justified? A multidisciplinary perspective from Africa and beyond. Journal of Sustainable Forestry 27 (3): 224-45.

Smith J, Colan V, Sabogal C y Snook L. 2006. Why policy reforms fail to improve logging practices: The role 
of governance and norms in Peru. Forest Policy and

Economics 8 (4): 458-69.

Tucker C. 2010. Learning on governance in forest ecosystems: Lessons from recent research. International Journal of the Commons 4 (2): 687.

Von Halle OB. 2014. Una nueva perspectiva para el análisis y calificación de la gobernanza forestal: Análisis de capacidades instaladas a nivel local para un manejo forestal legal y sostenible. En: Fanzeres A, Von Halle BO, Torres J, Orozco JMM, Mondragón M, Menton M, Bohórquez N, Aguirre N, Kometer R e Ingram V (eds.). Propuesta Metodológica para la Medición y Evaluación de la Gobernanza Forestal en Brasil, Colombia, Ecuador y Perú. TRAFFIC, Cambridge. Agroforestería
Esta investigación fue realizada por CIFOR como parte del Programa de Investigación de CGIAR sobre Bosques, Árboles y Agroforestería (FTA). El FTA es el programa de investigación para el desarrollo más grande del mundo, dedicado a mejorar el papel de bosques, árboles y la agroforestería para el desarrollo sostenible, la seguridad alimentaria, y frente al cambio climático. CIFOR dirige el programa FTA en asociación con Bioversity International, CATIE, CIRAD, ICRAF, INBAR y TBI.

La investigación del Programa FTA cuenta con el apoyo del Fondo Fiduciario del CGIAR: cgiar.org/funders avanzada, desarrollando las capacidades de sus socios y dialogando activamente con todos los actores involucrados, para informar sobre las políticas y las prácticas que afectan a los bosques y a las personas. CIFOR es un centro de investigación CGIAR y lidera su Programa de Investigación sobre Bosques, Árboles y Agroforestería (FTA por sus siglas en inglés). Nuestra sede central se encuentra en Bogor, Indonesia, y contamos con oficinas en Nairobi, Kenia; Yaundé, Camerún; Lima, Perú, y Bonn, Alemania. 